\title{
Effects of carbon and nitrogen sources on the antibacterial activity of Bacillus tequilensis BSM-F symbiotic with Halichondria panicea sponge from the cabbiya coast, Madura, Indonesia
}

\author{
Achmad Toto Poernomo, Sonia Khoirun Nisa, Zahratus Silmi Aliyah, Isnaeni* \\ Department of Pharmaceutical Chemistry, Faculty of Pharmacy, Universitas Airlangga, Surabaya \\ Jl. Dr. Ir. H. Soekarno, Mulyorejo, Surabaya, East Java, Indonesia
}

Accepted: 06-08-2020

\begin{abstract}
Sponges form obligate symbiotic associations with antibiotic-producing microorganisms. Many factors, including climate change, anthropogenic pollution, introduced species, and nutrient enrichment, have all been linked to the specific character of marine-symbiotic microorganisms. This recent study investigated the effects of carbon and nitrogen sources on the antibacterial activity of Bacillus tequilensis BSM-F in solid fermentation media, Zobell Marine Agar (ZMA), ZMA was added with various carbon and nitrogen sources at different concentrations to evaluate the effect of enrichment media on the antibacterial activity. The carbon sources used were glucose, glycerol, lactose, and starch, while the nitrogen sources were beef extract, soybean meal, malt extract, and casein. Each of which was added to ZMA at $0.5 \%, 1 \%, 1.5 \%$, and $2 \% \mathrm{w} / \mathrm{v}$. The inhibitory activity was denoted as the activity index, i.e., the ratio of the diameter of zone of inhibition to the diameter of the bacterial colony. B. tequilensis BSM-F exhibited the highest inhibitory activity against Staphylococcus aureus ATCC 25923 and Escherichia coli ATCC 25922 when cultured in media containing glycerol and casein. The optimum condition was achieved when glycerol and casein were each added to ZMA at $1.5 \% \mathrm{w} / \mathrm{v}$.
\end{abstract}

Keywords: Bacillus tequilensis BSM-F, carbon source, nitrogen source, inhibitory activity index

*Corresponding author:

Isnaeni

Department of Pharmaceutical Chemistry, Faculty of Pharmacy, Universitas Airlangga

Jl. Dr. Ir. H. Soekarno, Mulyorejo, Surabaya, East Java, Indonesia

Email: isna.yudi@gmail.com 


\section{INTRODUCTION}

Antibacterial compounds are broadly used for therapies in various countries, primarily because of their effectiveness in dealing with clinical problems, including the emergence of resistant pathogenic bacteria (Maestro and Sanz, 2007). For instance, Shigella has been reported to exhibit 50\% resistance to ampicillin, and Salmonella is resistant to ampicillin, chloramphenicol, and cotrimoxazole in $42 \%, 57 \%$, and $71 \%$ cases, respectively (Humaida, 2014). The discovery of new antibacterial compounds provides a solution to overcome resistance problems.

Microorganisms are a source of new antibacterials because they produce compounds fast and prolifically and have unlimited population size and straightforward control process (Davies and Davies, 2010). Microorganisms collected from the ocean are more superior compared to the ones originating in the land because the former has higher biodiversity and, consequently, produces more diverse antibacterial compounds than the latter (Abdelmohsen, 2014). There are more than 22,000 antibacterial metabolites produced by microorganisms, including bacteria and fungi (Subramani and Aalbersberg, 2012). According to (Long and Azam, 2001), bacteria that are symbiotic with marine organisms like sponges are known to produce higher antibacterial metabolites and have a broader spectrum than free-living bacteria.

The symbiotic relationship between Bacillus sp. and sponges has been found to affect the colonization of bacteria in the sponge through the production of antibacterial metabolites. The same relationship between Bacillus cereus QN03323 and the sponge Halichondria japonica produces YM-266183 and YM-266184 thiopeptide antibacterials that can inhibit the growth of Staphylococci and Enterococci (Nagai et al., 2003).

In bacteria, the biosynthetic reaction of secondary metabolites, including antibacterials, is influenced by the enzymes Polyketide Synthase (PKS) and Non-Ribosomal Peptide Synthetase (NRPS); which function as catalysts (Ansari et al., 2004). The performance of these enzymes depends on several factors, namely, temperature, $\mathrm{pH}$, incubation time, substrate concentration, inoculum concentration, and media. Therefore, the growing media needs to be added with nutrients, such as carbon and nitrogen sources, to produce antibacterial metabolites optimally.

This research was designed to analyze the effects of carbon and nitrogen addition on the production of antibacterial metabolites by sponge-symbiotic bacteria, Bacillus tequilensis BSM-F. The sponge Halichondria panicea was sampled from the Cabbiya Coast in Madura, Indonesia, because this coast is known to have highly diverse marine invertebrates, including $H$. panicea (Setiawan et al., 2018). The study was focused on producing the antibacterial metabolites in media containing carbon and nitrogen sources in 72 hours and examining their antibacterial activities against Staphylococcus aureus ATCC 25923 and Escherichia coli ATCC 25922.

\section{MATERIALS AND METHOD Materials}

Bacillus tequilensis BSM-F was isolated from the sponge Halichondria panicea collected from the Cabbiya Coast in Talango Sub-district, Sumenep District, Madura, at the coordinates $70^{0} 6^{\prime} 03.9$ "S and $113^{0} 58^{\prime} 33.6^{\prime \prime} \mathrm{E}$ and the depth of 3.5 meters below the sea level. The research materials also included Zobell Marine Agar (ZMA), carbon sources (glucose, lactose, glycerol, and starch) p.a., nitrogen sources (beef extract, soybean meal, malt extract, and casein) (Oxoid), nutrient agar (Oxoid), Staphylococcus aureus ATCC 25923 and Escherichia coli ATCC 25922 as the test bacteria, $\mathrm{NaCl}$ (E. Merck), and distilled water.

\section{Methods \\ Cultivation of Bacillus tequilensis BSM-F}

One Öse of colony was taken from B. tequilensis BSM-F isolates and swiped on the prepared media: $10 \mathrm{~mL}$ of ZMA (55.25 g dissolved in $1 \mathrm{~L}$ of distilled water), and then incubated at $37^{\circ} \mathrm{C}$ for 24 hours. The B. tequilensis BSM-F culture was stored at a temperature of $4^{\circ} \mathrm{C}$. 


\section{Fermentation of Bacillus tequilensis BSM-F}

B. tequilensis BSM-F was grown in a solid fermentation medium, ZMA, that had been added with carbon sources (glucose, lactose, glycerol, and starch, each at a concentration of $1 \%$ ) and nitrogen sources (beef extract, soybean meal, malt extract, and casein, each also at a concentration of $1 \%$ ). The ZMA was used as the control media, and each medium was prepared as much as 20 $\mathrm{mL}$. Then, a suspension of $B$. tequilensis BSM-F was created using the following procedures: 10 $\mathrm{mL}$ of $0.9 \%$ saline was added to the ZMA slant, vortexed until homogenous, measured, and adjusted to obtain the transmittance of $25 \%$ at $580 \mathrm{~nm}$. A total of $100 \mu \mathrm{L}$ of the suspension was removed using a micropipette, put into a test tube containing $20 \mathrm{~mL}$ of melted ZMA, vortexed, poured into a petri dish, and then incubated at $37^{\circ} \mathrm{C}$ for 72 hours.

\section{Antibacterial activity test}

The antibacterial activity was tested by the agar diffusion method with Nutrient Agar (NA) as the media. The NA media was prepared with $12 \mathrm{~mL}$ of a base layer and $8 \mathrm{~mL}$ of a seed layer. The base layer was poured first and allowed to solidify. Three $\mu \mathrm{L}$ of Staphylococcus aureus ATCC 25923 and Escherichia coli ATCC 25922 suspensions (25\% transmittance at $580 \mathrm{~nm}$ ) were added on the seed layer that was then poured into the petri dish containing the solidified base layer. For the antibacterial activity testing, the perforated colony in ZMA was cut and then placed on the surface of the NA media. After 72 hours of incubation at $37^{\circ} \mathrm{C}$, a clear area (zone of inhibition) formed around the colony was observed every 24 hours. Its diameter was measured with a caliper and presented in millimeters. The inhibitory activity was expressed as an activity index, i.e., the ratio of the diameters of the zone of inhibition to the bacterial colony in the agar media.

\section{Optimization of carbon and nitrogen sources}

For optimization, the carbon sources (glucose, lactose, glycerol, and starch) and nitrogen sources (beef extract, soybean meal, malt extract, and casein) were added to the solid fermentation media at various concentrations $(0.5,1.1 .5$, and $2 \%)$. The antibacterial activity was determined by the agar diffusion method, which was similar to the antibacterial activity test of the control media. The diameter of the zone of inhibition was measured every 24 hours with a caliper and presented in millimeters.

\section{Statistical analysis}

The data obtained were processed statistically by the Multivariate of Analysis (MANOVA) method in the Statistical Package for the Social Sciences (SPSS) v. 21.0 program. This test was intended to find a significant difference between the effects of carbon and nitrogen sources on the antibacterial activity.

\section{RESULTS AND DISCUSSION \\ Antibacterial activity test}

The growth inhibitory activity of Bacillus tequilensis BSM-F against Staphylococcus aureus ATCC 25923 was observed every 24 hours for six days at $37 \pm 1^{\circ} \mathrm{C}$. The inhibitory zones began to appear after 24 hours of incubation and increased until after 72 hours (Table 1). 
Table 1. The antibacterial activities of Bacillus tequilensis BSM-F against Staphyloccocus aureus ATCC 25923 (expressed as activity index)

\begin{tabular}{lcccccc}
\hline & \multicolumn{5}{c}{ Average Activity Index \pm SD } \\
\cline { 2 - 6 } & $\mathbf{2 4}$ hours & $\mathbf{4 8}$ hours & $\mathbf{7 2}$ hours & $\mathbf{9 6}$ hours & $\begin{array}{c}\text { 120 } \\
\text { hours }\end{array}$ & $\mathbf{1 4 4}$ hours \\
\hline ZMA & $1.12 \pm 0.01$ & $1.15 \pm 0.02$ & $1.16 \pm 0.02 *$ & $1.16 \pm 0.02$ & $1.16 \pm 0.02$ & $1.14 \pm 0.02$ \\
\hline ZMA + 1\% Glucose & $2.2 \pm 0.03$ & $2.22 \pm 0.03$ & $2.23 \pm 0.03$ & $2.23 \pm 0.03$ & $2.21 \pm 0.03$ & $2.19 \pm 0.04$ \\
\hline ZMA + 1\% Glycerol & $2.50 \pm 0.06$ & $2.53 \pm 0.08$ & $2.56 \pm 0.08 *$ & $2.56 \pm 0.08$ & $2.53 \pm 0.08$ & $2.51 \pm 0.08$ \\
\hline ZMA + 1\% Lactose & $1.50 \pm 0.02$ & $1.52 \pm 0.02$ & $1.53 \pm 0.02$ & $1.53 \pm 0.02$ & $1.53 \pm 0.02$ & $1.51 \pm 0.02$ \\
\hline ZMA + 1\% Amylum & $1.16 \pm 0.06$ & $1.19 \pm 0.04$ & $1.21 \pm 0.04$ & $1.21 \pm 0.04$ & $1.21 \pm 0.04$ & $1.19 \pm 0.05$ \\
\hline ZMA + 1\% Beef Extract & $1.32 \pm 0.06$ & $1.34 \pm 0.06$ & $1.35 \pm 0.06$ & $1.33 \pm 0.06$ & $1.33 \pm 0.06$ & ND \\
\hline $\begin{array}{l}\text { ZMA + 1\% Soybean } \\
\text { meal }\end{array}$ & $1.48 \pm 0.02$ & $1.49 \pm 0.02$ & $1.49 \pm 0.02$ & $1.48 \pm 0.02$ & $1.47 \pm 0.01$ & ND \\
\hline ZMA + 1\% Malt extract & $1.17 \pm 0.03$ & $1.19 \pm 0.02$ & $1.20 \pm 0.02$ & $1.20 \pm 0.02$ & $1.19 \pm 0.02$ & ND \\
\hline ZMA + 1\% Casein & $1.68 \pm 0.10$ & $1.69 \pm 0.10$ & $1.70 \pm 0.10 *$ & $1.69 \pm 0.09$ & $1.67 \pm 0.09$ & ND \\
\hline
\end{tabular}

*The highest activity index was first detected

The highest activity index was identified in media containing $1 \%$ glycerol and $1 \%$ casein after 72 -hour fermentation at $37 \pm 1{ }^{\circ} \mathrm{C}$, with the activity index of $2.56 \pm 0.08$ and $1.70 \pm 0.10$, respectively (Figure 1).
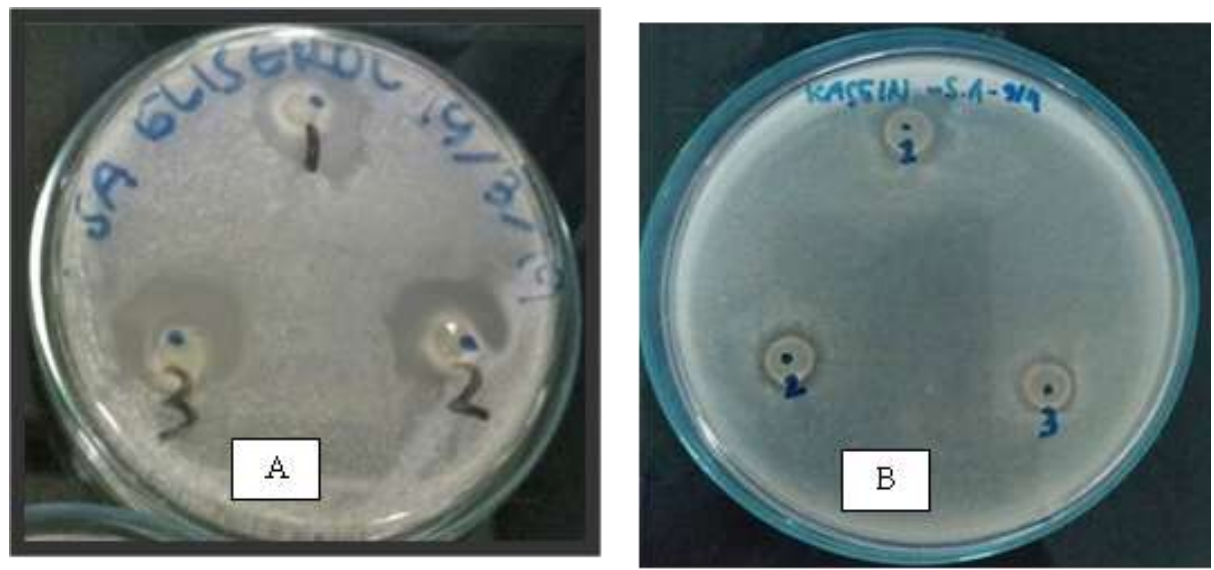

Figure 1. The antibacterial activities of Bacillus tequilensis BSM-F against Staphylococcus aureus ATCC on the test media containing $1 \%$ glycerol (A) and $1 \%$ casein (B)

The antibacterial activity of Bacillus tequilensis BSM-F against Escherichia coli ATCC 25922 was similar to that against Staphylococcus aureus (Table 2 and Figure 2). Identical inhibitory reactions were also observed after 24 and 72 hours of incubation. However, a difference was identified from the results of the MANOVA. 
Table 2. The antibacterial activities of Bacillus tequilensis BSM-F against Escherichia coli ATCC 25922 (expressed as activity index)

\begin{tabular}{lcccccc}
\hline \multirow{2}{*}{ Media } & \multicolumn{5}{c}{ Average Activity Index \pm SD } \\
\cline { 2 - 6 } ZMA & 24 hours & 48 hours & 72 hours & 96 hours & 120 hours & 144 hours \\
\hline ZMA + 1\% Glucose & $2.2 \pm 0.03$ & $2.22 \pm 0.03$ & $2.23 \pm 0.03$ & $2.23 \pm 0.03$ & $2.21 \pm 0.03$ & $2.19 \pm 0.04$ \\
\hline ZMA + 1\% Glycerol & $2.50 \pm 0.06$ & $2.53 \pm 0.08$ & $2.56 \pm 0.08 *$ & $2.56 \pm 0.08$ & $2.53 \pm 0.08$ & $2.51 \pm 0.08$ \\
\hline ZMA + 1\% Lactose & $1.50 \pm 0.02$ & $1.52 \pm 0.02$ & $1.53 \pm 0.02$ & $1.53 \pm 0.02$ & $1.53 \pm 0.02$ & $1.51 \pm 0.02$ \\
\hline ZMA + 1\% Amylum & $1.16 \pm 0.06$ & $1.19 \pm 0.04$ & $1.21 \pm 0.04$ & $1.21 \pm 0.04$ & $1.21 \pm 0.04$ & $1.19 \pm 0.05$ \\
\hline ZMA + 1\% Beef Extract & $1.30 \pm 0.05$ & $1.31 \pm 0.06$ & $1.32 \pm 0.06$ & $1.31 \pm 0.06$ & $1.31 \pm 0.07$ & ND \\
\hline $\begin{array}{l}\text { ZMA + 1\% Soybean } \\
\text { meal }\end{array}$ & $1.55 \pm 0.07$ & $1.56 \pm 0.07$ & $1.58 \pm 0.07$ & $1.56 \pm 0.07$ & $1.55 \pm 0.07$ & ND \\
\hline ZMA + 1\% Malt extract & $1.22 \pm 0.05$ & $1.23 \pm 0.05$ & $1.24 \pm 0.05$ & $1.23 \pm 0.05$ & $1.23 \pm 0.05$ & ND \\
\hline ZMA + 1\% Casein & $1.83 \pm 0.14$ & $1.84 \pm 0.14$ & $1.85 \pm 0.14 *$ & $1.84 \pm 0.14$ & $1.83 \pm 0.14$ & ND \\
\hline
\end{tabular}

*The highest activity index was first detected

In optimum conditions, the antibacterial activities of $B$. tequilensis BSM-F against Staphylococcus aureus and Escherichia coli were higher than on ZMA. This result implies that carbon and nitrogen sources positively affect the antibacterial activities of B. tequilensis BSM-F. For future research, these optimum conditions can be implemented on pathogens as the test microorganisms.

The MANOVA revealed that $1 \%$ glycerol (carbon source) and $1 \%$ casein (nitrogen source) produced the most considerable effects on the antibacterial activities of B. tequilensis (Sig. $=0.000<0.05)$.
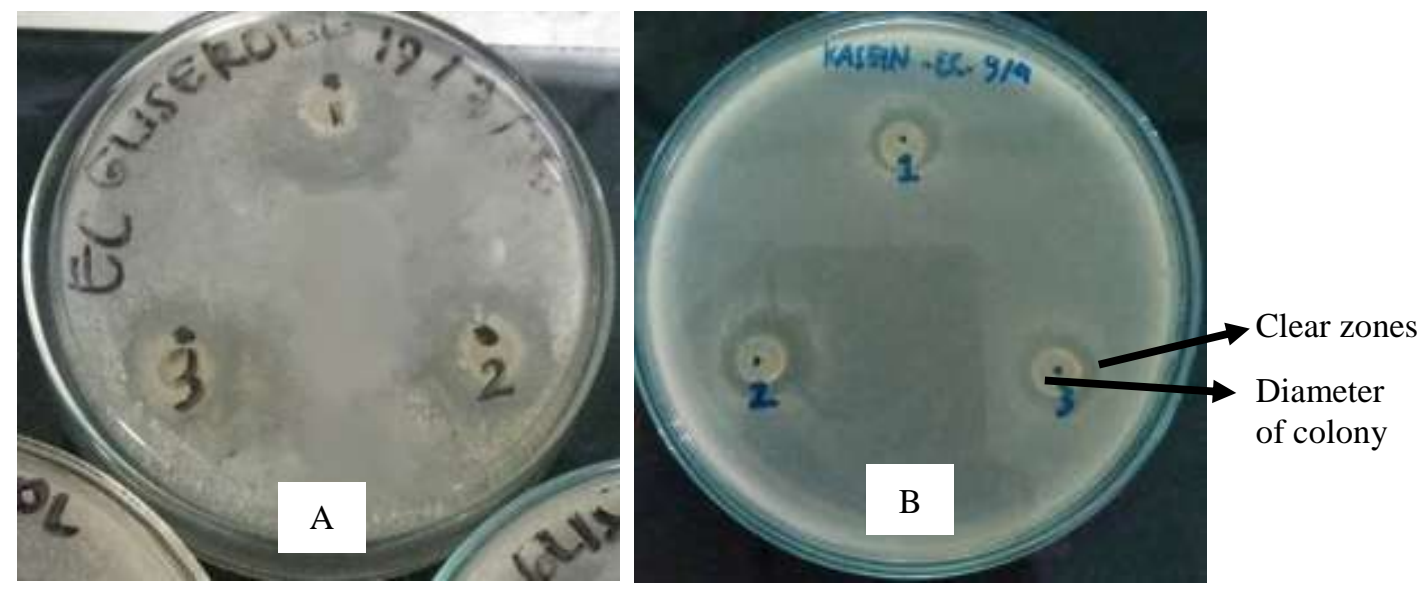

Figure 2. The antibacterial activities of Bacillus tequilensis BSM-F against Escherichia coli ATCC 25922 in the test media containing $1 \%$ glycerol (A) and $1 \%$ casein (B) 


\section{Optimized glycerol and casein concentrations}

Bacillus tequilensis BSM-F grown on ZMA added with various concentrations of glycerol and casein exhibited varying levels of antibacterial activities (Figure 3 and Figure 4). The highest antibacterial activity was obtained at a concentration of $1.5 \%$ and incubation temperature of $37^{\circ} \mathrm{C}$.

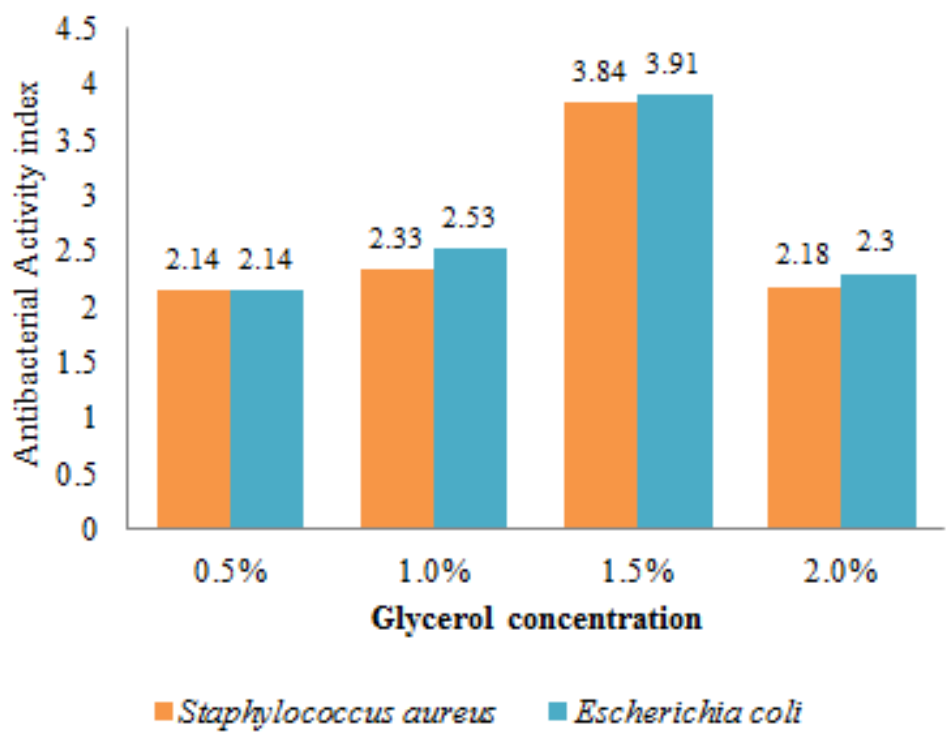

Figure 3. The inhibitory activities of Bacillus tequilensis BSM-F on various concentrations of glycerol against Staphylococcus aureus ATCC 25923 and Escherichia coli ATCC 25922 after six hours of fermentation

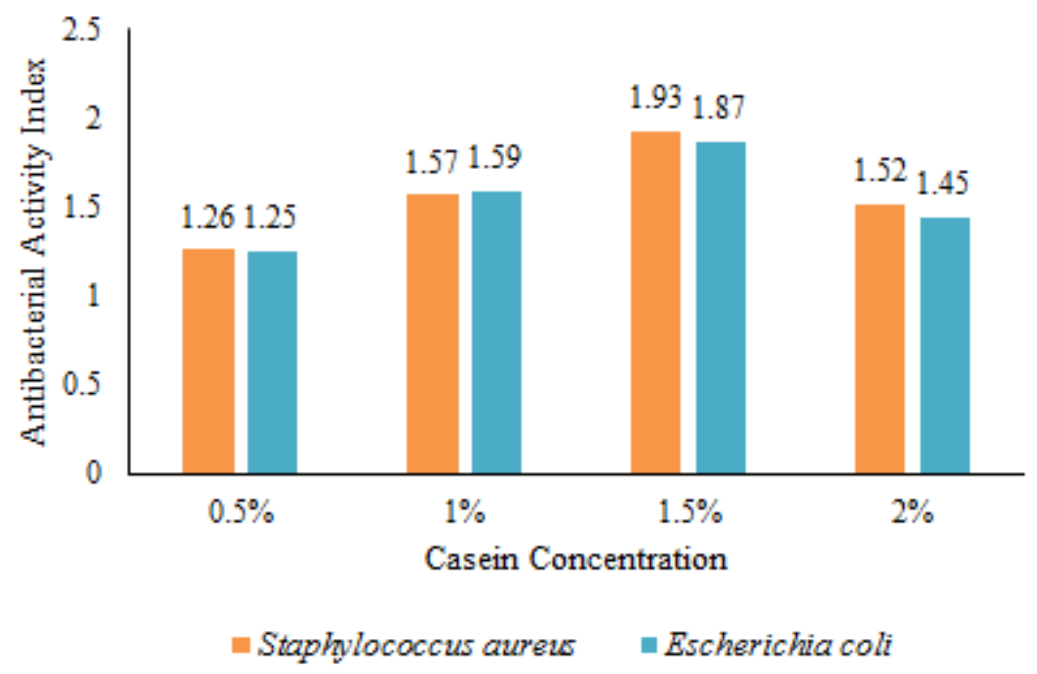

Figure 4. The antibacterial activities of Bacillus tequilensis BSM-F on various concentrations of casein against Staphylococcus aureus ATCC 25923 and Escherichia coli ATCC 25922 after six hours of fermentation

Based on Figure 3 and Figure 4, the various concentrations of glycerol and casein added to ZMA caused different antibacterial activities. The optimum concentrations of glycerol and casein 
were determined based on the most significant antibacterial activities that Bacillus tequilensis BSM-F produced, namely, $1.5 \%$ glycerin and $1.5 \%$ casein $($ Sig. $=0.000<0.05$ ).

This study was designed to determine the optimum condition for the production of antibacterial metabolites from Bacillus tequilensis by regulating the addition of carbon and nitrogen sources to the production media. ZMA was chosen because of its nutrient content; it is rich in minerals and salts to support the growth of marine bacteria and their metabolites production. A previous study has reported that Zobell Marine Agar can grow and produce antibacterial metabolites from Bacillus tequilensis symbiotic with a sponge (Kiran et al., 2018).

The fermentation of Bacillus tequilensis BSM-F in solid media is profitable for activity screening purposes, but for metabolite production, this technique has limitations. It allows contact between nutrients in the media and bacterial cells with adequate oxygen supply, and the process is considered fast and straightforward because it does not require special tools. The role of ZMA in metabolite production can be optimized by adding external nutrients like carbon sources for biomass formation, growth nutrition, and increased biosynthetic energy of metabolites (Mcneil and Harvey, 2008). Carbon sources used in this research were monosaccharides (glycerol and glucose), disaccharides (lactose), and polysaccharides (starch). Among the four carbons, glycerol, as polyvalent alcohol, has a simpler structure that can be disintegrated more efficiently into an energy source. Glycerol is also commonly used as a storage medium for microorganisms at low temperatures. Nitrogen sources are also needed in the synthesis of proteins, DNA, RNA, and ATP as energy storage and transfer in bacterial cells (Tortora and Derrickson, 2018). Except for casein, the nitrogen sources in this study were derived from complex substances, such as beef extract, soybean meal, and malt extract. Meanwhile, casein is a protein that is commonly used together with soybean as media, consists of eight amino acids, and has a simpler structure than other nitrogen sources.

Due to the formation of dihydroxyacetone phosphate (DHAP), the NADH regeneration occurs in the glycerol substrate, which can suppress acetate as a by-product and accumulate pyruvate. While pyruvate accumulates in glycerol substrate, glucose is the opposite. Pyruvate can accumulate due to available NADH and aerobic conditions (Yazdani and Gonzalez, 2007). It is a precursor of amino acids that compose the NRPS and PKS enzymes, which catalyze secondary metabolites into antibacterial agents. Meanwhile, according to Kampen, casein has a fairly high nitrogen content (13.5\%) and various amino acids (Kampen, 2014). These amino acids are later transformed into a substrate in the biosynthetic process of PKS and NRPS enzymes, which is responsible for producing secondary metabolites, including antibacterials (Desriac et al., 2013).

Based on the preliminary research, bacterial growth has reached a stationary phase; therefore, the production of antibacterial metabolites is potentially high. The antibacterial metabolites from Bacillus tequilensis BSM-F can be produced in media added with various carbon and nitrogen sources in 72 hours.

The antibacterial activities of Bacillus tequilensis BSM-F were tested by agar diffusion with Nutrient Agar (base and seed layers) as the media. A modified technique was performed by perforating and cutting the agar culture containing Bacillus tequilensis BSM-F and placing it on the surface of the seed layer for inhibitory activity evaluation. The diffusion method is simple and straightforward (it does not require special tools). The other advantages include the ability to test large numbers of bacteria and antibacterial agents and the ease of result interpretation. During the observation, a clear zone was formed around the agar colony, indicating bacterial growth inhibition. The longer the diameter of the zone of inhibition, the higher the antibacterial activity tested (Bonev et al., 2008). The diameter was measured using a caliper, with a precision of 0.05 $\mathrm{mm}$, and inputted to the antibacterial activity index formula, i.e., a ratio of the diameter of the zone of inhibition to the diameter of the bacterial colony. 
In two types of media (containing carbon and nitrogen sources), zones of inhibition began to appear after 24 hours of incubation. The diameter was formed the largest on Day 3 before it started to shrink because the Bacillus tequilensis BSM-F tested was on the initial phase of death. Bacillus tequilensis $\mathrm{BSM}-\mathrm{F}$ grown in $\mathrm{ZMA}+1 \%$ glycerol and $\mathrm{ZMA}+1 \%$ casein were found to exhibit the most potent antibacterial activities against Staphylococcus aureus ATCC 25923 and Escherichia coli ATCC 25922. Based on the MANOVA results, the mean difference between ZMA+1\% glycerol and $\mathrm{ZMA}+1 \%$ casein was the largest among the carbon and nitrogen sources, and the subset value was in a different column.

The additions of glycerol and casein to the fermentation media were optimized by testing various concentrations: $0.5 \%, 1 \%, 1.5 \%$, and $2 \%$. This range was selected based on (Haddar et al., 2007 ) that used glycerol at these concentrations to examine the antibacterial activities of bacitracin and found that $0.5 \%$ was the smallest concentration of glycerol to produce secondary metabolites. In other words, at lower concentrations, glycerin will not be able to produce secondary metabolites. When the levels added were raised to $1-1.5 \%$, there was an increase in the production of secondary metabolites. However, at $2 \%$ and above, the secondary metabolites produced experienced a decrease, which is assumed to be the effect of feedback inhibition.

When cultured in fermentation media containing ZMA $1.5 \%$ glycerol (carbon sources) and $\mathrm{ZMA}+1.5 \%$ casein (nitrogen sources), Bacillus tequilensis BSM-F exhibited optimum antibacterial activities against Staphylococcus aureus ATCC 25923 and Escherichia coli ATCC 25922. The MANOVA also revealed that the mean difference between $1.5 \%$ glycerol and $1.5 \%$ casein was the most significant among the tested concentrations of the carbon and nitrogen sources, and the subset value was in a different column.

An increase in antibacterial activities does not always follow a higher concentration of added carbon and nitrogen sources. At some point, the test bacteria can experience saturation with the conditions of their media. Due to the excess nutrient content, there are more dead bacteria than the alive ones in the culture.

\section{CONCLUSION}

The optimum antibacterial activity of Bacillus tequilensis BSM-F against Staphylococcus aureus ATCC 25923 and Escherichia coli ATCC 25922 can be obtained by culturing them in a solid fermentation media containing $1.5 \%$ glycerol or $1.5 \%$ casein.

\section{REFERENCES}

Abdelmohsen, U. R. (2014). Diversity, abundance and natural products of marine spongeassociated actinomycetes. Natural Product Reports, 31(3), 381-399. https://doi.org/10.1039/c3np70111e

Ansari, M. Z., Yadav, G., Gokhale, R. S., \& Mohanty, D. (2004). NRPS-PKS: A knowledge-based resource for analysis of NRPS-PKS megasynthases. Nucleic Acids Research, 32(WEB SERVER ISS.), 405-413. https://doi.org/10.1093/nar/gkh359

Bonev, B., Hooper, J., \& Parisot, J. (2008). Principles of assessing bacterial susceptibility to antibiotics using the agar diffusion method. Journal of Antimicrobial Chemotherapy, 61(6), 1295-1301. https://doi.org/10.1093/jac/dkn090

Davies, J., \& Davies, D. (2010). Origins and evolution of antibiotic resistance. Microbiology and Moleculer Biology Reviews, 74(3), 417-433.

Desriac, F., Jégou, C., Balnois, E., Brillet, B., Le Chevalier, P., \& Fleury, Y. (2013). Antimicrobial peptides from marine proteobacteria. Marine Drugs, 11(10), 3632-3660. https://doi.org/10.3390/md11103632.

Haddar, H. O., Aziz, G. M., \& Al-Gelawi, M. H. (2007). Optimization of bacitracin production by Bacillus licheniformis B5. Pakistan Journal of Biological Science, 10, 972-976.

Humaida, R. (2014). Strategy to Handle Resistance of Antibiotics. Majority Medical Journal of 
Lampung University, 3(7), 1-8.

Kampen, W. H. (2014). Nutritional requirements in fermentation processes. In Fermentation and Biochemical Engineering Handbook (pp. 37-57). Elsevier.

Kiran, G. S., Priyadharsini, S., Sajayan, A., Ravindran, A., \& Selvin, J. (2018). An antibiotic agent pyrrolo [1, 2-a] pyrazine-1, 4-dione, hexahydro isolated from a marine bacteria Bacillus tequilensis MSI45 effectively controls multi-drug resistant Staphylococcus aureus. Royal Society of Chemistry Advances, 8(32), 17837-17846.

Long, R. A., \& Azam, F. (2001). Antagonistic interactions among marine pelagic bacteria. Applied and Environmental Microbiology, 67(11), 4975-4983.

Maestro, B., \& Sanz, J. M. (2007). Novel approaches to fight Streptococcus pneumoniae. Recent Patents on Anti-Infective Drug Discovery, 2(3), 188-196.

Mcneil, B., \& Harvey, L. M. (2008). Practical Fermentation Technology. Practical Fermentation Technology. John Wiley \& Sons Ltd, The Atrium, Southern Gate, Chichester, West Sussex PO19 8SQ, England.

Nagai, K., Kamigiri, K., Arao, N., Suzumura, K.-I., Kawano, Y., Yamao, M., ... Suzuki, K. (2003). YM-266183 and YM-266184, Novel thiopeptide antibiotics produced by bacillus cereus isolated from a marine sponge. The Journal of Antibiotics, 56(2), 123-128.

Setiawan, E., Kamal, F., \& Ashuri, M. (2018). Shallow water sponges that associated to mangrove ecosystem at Labuhan conservation area in Sepulu , Bangkalan , Madura , East Java Province. NICHE Journal of Tropical Biology, 1(2), 19-29.

Subramani, R., \& Aalbersberg, W. (2012). Marine actinomycetes: An ongoing source of novel bioactive metabolites. Microbiological Research (Vol. 167). Elsevier GmbH. https://doi.org/10.1016/j.micres.2012.06.005.

Tortora, G. J., \& Derrickson, B. H. (2018). Principles of anatomy and physiology. John Wiley \& Sons.

Yazdani, S. S., \& Gonzalez, R. (2007). Anaerobic fermentation of glycerol: a path to economic viability for the biofuels industry. Current Opinion in Biotechnology, 18(3), 213-219. 
